\title{
Editorial: Peripheral Immunity in Parkinson's Disease: Emerging Role and Novel Target for Therapeutics
}

\author{
Cristoforo Comi ${ }^{1 *}$, Marco Cosentino ${ }^{2}$ and Rodrigo Pacheco ${ }^{3}$ \\ ${ }^{1}$ Neurology Unit, Department of Translational Medicine, Interdisciplinary Research Center of Autoimmune Diseases, \\ Movement Disorders Center, University of Piemonte Orientale, Novara, Italy, ${ }^{2}$ Center of Research in Medical Pharmacology, \\ University of Insubria, Varese, Italy, ${ }^{3}$ Laboratory of Neuroimmunology, Fundación Ciencia and Vida, Nuñoa, Departamento de \\ Ciencias Biológicas, Facultad de Ciencias de la Vida, Universidad Andres Bello, Santiago, Chile
}

Keywords: immunity, alpha-synucein, gut-brain axis, T cell, probiotics

\section{Editorial on the Research Topic}

\section{Peripheral Immunity in Parkinson's Disease: Emerging Role and Novel Target for Therapeutics}

Parkinson's disease (PD) is the second most common neurodegenerative disease, affecting up to 10 million people worldwide. PD has no cure yet, and patients rely only on symptomatic treatments. The hallmarks of PD are progressive loss of dopaminergic neurons in the substantia nigra, appearance of intracellular inclusions of aggregated $\alpha$-synuclein ( $\alpha$-syn), called Lewy bodies (LB), and neuroinflammation resulting from microglia activation $(1,2)$. Understanding the causes of neurodegeneration in PD remains, so far, a challenging goal. Nevertheless, the recent identification of the involvement of peripheral immunity is raising increasing interest, as it may provide unprecedented opportunities to better understand PD pathogenesis, to identify clinically meaningful biomarkers and hopefully also novel therapeutic strategies $(3,4)$.

Besides a strong genetic association between the major histocompatibility class II locus and PD risk $(5,6)$, evidence supporting the role of peripheral immunity in PD include more rapid PD progression in the presence of a pro-inflammatory cytokine profile in the blood (7), a Th1biased CD4 $+\mathrm{T}$ cell profile $(8,9)$, as well as an altered CD8 $+\mathrm{T}$ cell profile, with increased activation and reduced senescence markers (10). Remarkably, $\alpha$-syn may trigger infiltration into the brain of $\mathrm{T}$ cells which in turn contribute to exacerbation of $\alpha$-syn pathology, neurotoxicity and neurodegeneration (11-13), and the reported ability of T cells from PD patients to generate an autoimmune response to $\alpha$-syn $(14,15)$ is even leading to reconsider PD as an autoimmune disorder (16).

Despite all this knowledge, however, the nature of immune dysregulation in PD, its relationship with neuroinflammation and neurodegeneration in the brain, the changes in peripheral immunity during disease progression, and whether targeting peripheral immunity may be beneficial to PD patients, all remain to be established. This Research Topic has been launched with the aim to collect high-quality and state-of-the-art articles covering all the aspects relevant to the relationship between immunity and neurodegeneration in PD, and bringing together research teams from different but synergistic scientific fields.

The 12 articles, seven review and five original articles, that were finally accepted for publication, offer the opportunity to explore the most relevant aspects of this emerging Research Topic. Of course, review articles discuss the contribution of immunity and inflammation to PD pathogenesis in a more general framework. This is the case of Caggiu et al., who focus on evidence linking infections and abnormal protein accumulation to immune system activation and critically discuss the possibility that autoimmunity may take part in PD pathogenesis. Fuzzati-Armentero et al. make an interesting parallel between PD patients and toxin-induced animal models, discussing differences and similarities in the context of neuroinflammation and immune responses, 
including the potential to guide novel therapeutic strategies. Troncoso Escudero et al. provide an updated perspective on the complex dynamics which orchestrate immune responses bothinside the central nervous system (CNS) and in the periphery. In this context, authors also discuss the potential targeting of astrocytes and microglia, as well as gut microbiome, with their therapeutic implications in PD.

García-González et al. review the involvement of stress signaling by the endoplasmic reticulum, which is crucially involved in protein aggregation and proteostasis dysfunction. Authors discuss how such signaling impacts on brain-associated immune cells and the possible implications to neuroinflammation and development of neurodegenerative diseases.

Scott et al. focus on the role of $\alpha$-syn autoantibodies in PD, showing that there is weak evidence for an increase in $\alpha$-syn auto-antibodies in PD patients particularly in early disease phase, but also underlining that more evidence is needed to support a robust relationship.

Finally, two reviews point to the role of T cells. Campos-Acuña et al. critically discuss the possibility that $\mathrm{T}$ cell driven inflammation, which has a crucial role in dopaminergic degeneration in $\mathrm{PD}$, is triggered in the gut mucosa. Accordingly, they show how structural components of commensal bacteria and/or mediators produced by gut-microbiota, including shortchain fatty acids and dopamine, may affect the behavior of $\mathrm{T}$ cells, triggering the development of $\mathrm{T}$ cell responses against LB, initially confined to the gut mucosa but later extended to the brain. Storelli et al. summarize the current knowledge on the contribution of Th17 cells and IL-17 in PD, also assessing their therapeutic relevance. They underline that both animal and clinical studies are limited. Only a few studies provide mechanistic evidence and none of them investigates the eventual relationship between Th17/IL-17 and clinically relevant endpoints.

As regards original articles, Peralta Ramos et al. investigated the role of peripheral immune cells in the spreading of $\alpha$ syn strains to the CNS. Authors provide evidence that $\alpha$ syn administration in mice can induce microglia activation and leukocytes recruitment toward the CNS. Monocytes primed by intraperitoneal LPS administration internalize $\alpha$-syn, with subsequent CNS dissemination. In addition, the $\alpha$-syn ribbons strain determines differential recruitment of CD4+ and CD8+ T cells.

Elgueta et al. explore the role of dopamine receptor D3 (DRD3) signaling in peripheral blood CD4+ T cells from PD patients. They find that immune phenotypes favored by DRD3 signaling, namely Th1 and Th17 cells, are increased in the peripheral blood cells of PD patients compared to controls.

Further, they support their findings by selective DRD3antagonism in this subset of lymphocytes in parkinsonian mice, obtaining a therapeutic effect on motor impairment.

Magistrelli et al. studied the effects of an in vitro challenge with probiotic bacterial strains to peripheral blood mononuclear cells (PBMCs) of PD patients and controls. All strains inhibited inflammatory cytokines and ROS production in both patients and controls, but most strikingly Lactobacillus salivarius and acidophilus. Furthermore, most strains restored the integrity of an artificial membrane model integrity and inhibited Escherichia coli and Klebsiella pneumoniae overgrowth. Finally, authors showed that the studied strains did not express tyrosine decarboxylase genes, which are known to decrease levodopa bioavailability.

Wijeyekoon et al. assessed monocyte functions in early-moderate PD compared to age and gendermatched controls. They found that PD monocytes display enhanced phagocytosis, but no significant differences in migration or cytokine secretion compared to controls.

White et al. investigated cell-extrinsic factors in systemic immune activation by using $\alpha$-syn monomers and fibrils, as well as bacterial toxins, to stimulate PBMCs from PD patients and controls. They found no differences in cytokine production, nor in mRNA expression in patients vs. controls. By contrast, $\alpha$-syn monomers increased production of IL- $1 \beta$ and IL-18 to levels significantly increased compared to those induced by lowlevel endotoxin.

In conclusion, this Research Topic provides a comprehensive overview of our current understanding of how adaptive and innate immune systems in the periphery are affected by infectious agents, commensal bacteria and pathogenic forms of $\alpha$-syn, triggering an immune response in the central nervous system, possibly targeted at endogenous neoantigens such as $\alpha$-syn itself, which eventually feeds neuroinflammation and neurodegeneration. Despite all this knowledge, however, much research is still required to establish the nature of immune dysregulation occurring in $\mathrm{PD}$, how the immune system is involved in the prodromal phases of $\mathrm{PD}$, and whether targeting peripheral immunity may favorably affect disease progression. We strongly hope that this collection of articles, providing a new insight of the physiopathology of PD, will encourage more laboratory and clinical research leading to the development of novel immunotherapeutics and probiotics as treatments of this disorder.

\section{AUTHOR CONTRIBUTIONS}

All authors listed have made a substantial, direct and intellectual contribution to the work, and approved it for publication.

\section{FUNDING}

The authors gratefully acknowledge the support provided by: Fondazione Cariplo (Progetto 2011-0504); Fondazione Unione Banche Italiane per Varese onlus (grant 18/07/2017); the AGING Project, Department of Excellence, Università degli Studi del Piemonte Orientale; Programa de Apoyo a Centros con Financiamiento Basal AFB-170004 from Comisión Nacional de Investigación Científica y Tecnológica de Chile (CONICYT); grant FONDECYT-1170093 from Fondo Nacional de Desarrollo Científico y Tecnológico de Chile; grant MJFF-10332.01 and MJFF-15076 (to RP) from Michael J. Fox Foundation for Parkinson Research. All these grant programs contributed to the development of the ideas and of the experimental research reviewed in the text. 


\section{REFERENCES}

1. Eriksen JL, Wszolek Z, Petrucelli L. Molecular pathogenesis of Parkinson disease. Arch Neurol. (2005) 62:353-7. doi: 10.1001/archneur.62.3.353

2. Dehay B, Bourdenx M, Gorry P, Przedborski S, Vila M, Hunot S, et al. Targeting $\alpha$-synuclein for treatment of Parkinson's disease: mechanistic and therapeutic considerations. Lancet Neurol. (2015) 14:855-66. doi: 10.1016/S1474-4422(15)00006-X

3. Mosley RL, Hutter-Saunders JA, Stone DK, Gendelman HE. Inflammation and adaptive immunity in Parkinson's disease. Cold Spring Harb Perspect Med. (2012) 2:a009381. doi: 10.1101/cshperspect.a009381

4. Sommer A, Winner B, Prots I. The Trojan horse - neuroinflammatory impact of T cells in neurodegenerative diseases. Mol Neurodegener. (2017) 12:78. doi: 10.1186/s13024-017-0222-8

5. Saiki M, Baker A, Williams-Gray CH, Foltynie T, Goodman RS, Taylor CJ, et al. Association of the human leucocyte antigen region with susceptibility to Parkinson's disease. J Neurol Neurosurg Psychiatry. (2010) 81:8901. doi: $10.1136 /$ jnnp.2008.162883

6. International Parkinson Disease Genomics Consortium, Nalls MA, Plagnol V, Hernandez DG, Sharma M, Sheerin UM, et al. Imputation of sequence variants for identification of genetic risks for Parkinson's disease: a meta-analysis of genome-wide association studies. Lancet. (2011) 377:6419. doi: 10.1016/S0140-6736(10)62345-8

7. Williams-Gray $\mathrm{CH}$, Wijeyekoon R, Yarnall AJ, Lawson RA, Breen DP, Evans JR, et al. Serum immune markers and disease progression in an incident Parkinson's disease cohort (ICICLE-PD). Mov Disord. (2016) 31:9951003. doi: $10.1002 / \mathrm{mds} .26563$

8. Kustrimovic N, Rasini E, Legnaro M, Bombelli R, Aleksic I, Blandini F, et al. Dopaminergic receptors on $\mathrm{CD} 4+\mathrm{T}$ naive and memory lymphocytes correlate with motor impairment in patients with Parkinson's Disease. Sci Rep. (2016) 6:33738. doi: 10.1038/srep33738

9. Kustrimovic N, Comi C, Magistrelli L, Rasini E, Legnaro M, Bombelli $\mathrm{R}$, et al. Parkinson's disease patients have a complex phenotypic and functional Th1 bias: cross-sectional studies of CD4+ Th1/Th2/T17 and Treg in drug-naïve and drug-treated patients. J Neuroinflammation. (2018) 15:205. doi: 10.1186/s12974-018-1248-8
10. Williams-Gray CH, Wijeyekoon RS, Scott KM, Hayat S, Barker RA, Jones JL. Abnormalities of age-related T cell senescence in Parkinson's disease. $J$ Neuroinflammation. (2018) 15:166. doi: 10.1186/s12974-018-1206-5

11. Ferreira SA, Romero-Ramos M. Microglia response during Parkinson's Disease: alpha-synuclein intervention. Front Cell Neurosci. (2018) 12:247. doi: 10.3389/fncel.2018.00247

12. Sommer A, Fadler T, Dorfmeister E, Hoffmann AC, Xiang W, Winner $\mathrm{B}$, et al. Infiltrating $\mathrm{T}$ lymphocytes reduce myeloid phagocytosis activity in synucleinopathy model. J Neuroinflammation. (2016) 13:174. doi: 10.1186/s12974-016-0632-5

13. Sommer A, Maxreiter F, Krach F, Fadler T, Grosch J, Maroni M, et al. Th17 lymphocytes induce neuronal cell death in a human iPSCbased model of Parkinson's Disease. Cell Stem Cell. (2018) 23:12331.e6. doi: 10.1016/j.stem.2018.06.015

14. Garretti F, Agalliu D, Lindestam Arlehamn CS, Sette A, Sulzer D. Autoimmunity in Parkinson's Disease: the role of $\alpha$-Synuclein-specific $\mathrm{T}$ cells. Front Immunol. (2019) 10:303. doi: 10.3389/fimmu.2019. 00303

15. Sulzer D, Alcalay RN, Garretti F, Cote L, Kanter E, Agin-Liebes J, et al. T cells from patients with Parkinson's disease recognize alpha-synuclein peptides. Nature. (2017) 546:656-61. doi: 10.1038/nature22815

16. Bandres-Ciga S, Cookson MR. Alpha-synuclein triggers T-cell response. Is Parkinson's disease an autoimmune disorder? Mov Disord. (2017) 32:1327.doi: $10.1002 / \mathrm{mds} .27116$

Conflict of Interest: The authors declare that the research was conducted in the absence of any commercial or financial relationships that could be construed as a potential conflict of interest.

Copyright (๑) 2019 Comi, Cosentino and Pacheco. This is an open-access article distributed under the terms of the Creative Commons Attribution License (CC BY). The use, distribution or reproduction in other forums is permitted, provided the original author(s) and the copyright owner(s) are credited and that the original publication in this journal is cited, in accordance with accepted academic practice. No use, distribution or reproduction is permitted which does not comply with these terms. 